\title{
Performance Evaluation of Petri Nets Execution Algorithms
}

\author{
Ramón Piedrafita Moreno, José Luis Villarroel Salcedo \\ Aragón Institute for Engineering Research (I3A), University of Zaragoza, \\ Maria de Luna, 1. 50018, Zaragoza, Spain \\ \{piedrafi,jlvilla\}@unizar.es
}

\begin{abstract}
In this paper we present a performance evaluation of interpreted and centralized implementation techniques of Petri nets. These implementation techniques allow the translation of a system model expressed by a Petri net to an actual system with the same behaviour as the model. It has been used in different application fields such as programmable logic controllers. Four techniques have been analysed: Brute Force, Enabled Transitions, Static Representing Places and Dynamic Representing Places. The analysis has been carried out over a Petri net library composed by well know models which can be scaled using a parameter. The analysis of results shows that the performance of the algorithms depends on the Petri net behaviour (concurrency v.s. effective conflicts). However, it is also showed that Brute Force technique can be discarded and that the Static Representing Places performs better that the dynamic version when the selection of representing places is done based on behavioural information.
\end{abstract}

\section{INTRODUCTION}

$\mathrm{P}$ etri nets $(\mathrm{PN})$ are a formalism which is well suited for modelling concurrent discrete event systems: it has been satisfactorily applied in fields such as communication networks, computer systems, discrete part manufacturing systems, etc. Net models are often regarded as self documented specifications, because their graphical nature facilitates communication among designers and users. Petri Nets have a strong mathematical foundation which allows the validation and verification of a wide set of correctness and liveness properties. Moreover, these models are executable and can be used to animate and simulate the behaviour of the system and can also be used for monitoring purposes once the system is readily working. The final system can be derived from a Petri net model by means of hardware and software (code generation) implementation techniques. In other words, they can be used all along in the life cycle of a system. In this paper we assume that the reader knows the basic concepts of Petri Nets[1].

The implementation of Petri Nets has received considerable attention from researchers over the last twenty five years. The implementation is the translation of a system model expressed by a Petri net to an actual system with the same behaviour as the model. It has been studied in numerous application fields such as programmable logic controllers, digital hardware, simulation, robotic systems and other concurrent software applications.

This work was funded by the NERO project DPI2006-07928 of the Spanish Ministry of Science and Technology.
A Petri net implementation can be hardware or software. However we are interested in the second approach, the software implementation. A software implementation is a program which fires the net transitions, observing marking evolution rules, i.e., plays the "token game". An implementation is composed of a control part and an operational part. The control part corresponds to the structure, marking and evolution rules of the Petri net. On the other hand, the operational part is the set of actions and/or codes of the application, associated with the net elements.

According to different criteria, a Petri net implementation can be mainly classified as compiled or interpreted, as sequential or concurrent and as centralized or decentralized.

An implementation is interpreted if the net structure and the marking are codified as data structures. These data structures are used by one or more tasks called interpreters to make the net evolve. The interpreters do not depend on the implemented net. A compiled implementation is based on the generation of one or more tasks whose control flow corresponds to net evolutions.

A sequential implementation is composed of only one task, even in nets with concurrency. This kind of implementation is common in applications whose operational part is composed by impulsional actions without significant execution time. This is the case of programmable logic controllers, see for example [2] or [3]. The research effort in this field produced an important result, the Grafcet [4], a standardized language for programmable logic controllers. A concurrent implementation is composed by a set of tasks whose number is equal to or greater than the actual concurrency of the net. Examples of concurrent implementations can be seen in [5] or in [6].

In a centralized implementation the full control part is executed by just one task, commonly called token player or coordinator. The operational part of the implementation can be distributed in a set of tasks to guarantee the concurrence expressed by the net (see for example [5]). Thus, the coordinator acts as the operating system kernel of a multitasking application. To characterize a centralized implementation, the algorithm is important to determine which transitions are enabled and can fire. Apart of the simple exhaustive test of all transitions, there are various solutions to reduce the costs of the enabling test.

The main objective of the current paper is to evaluate the current interpreted and centralized implementation techniques of Petri nets from the point of view of the overload introduced for the control part. For this reason we have implemented different $\mathrm{PN}$ execution algorithms which will be 
executed within an extensive size-scalable Petri nets library. In this work we will show that the computing time of the control part depends greatly on the PN structure and on the algorithm chosen to execute it. We will analyse the response time of the algorithms and propose techniques which will allow us to choose the most suitable algorithm to execute a $\mathrm{PN}$. The goal is to minimise the computing time of the control part and in this manner minimise the controller reaction time.

The organization of this paper is as follows. In Section II, we review the centralized implementation techniques for PN. The description of test are show in Section III. Section IV shows the results of the performance evaluation for the different implementation techniques. Finally, in section V, we present the main conclusions and suggest future lines of research.

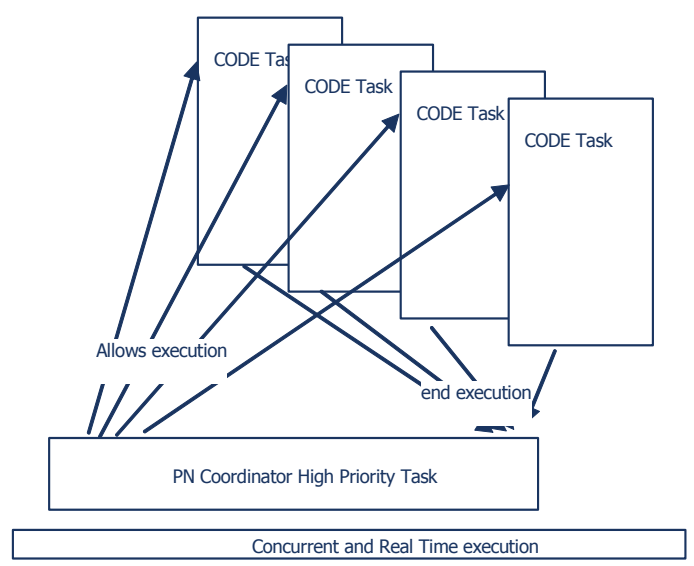

Fig. 1. Coordinator and CODE task execution.

\section{CENTRALISED IMPLEMENTATION: STRUCTURE AND ALGORITHMS}

An implementation of a Petri net has a strong dependency on the interpretation of the net model, namely, how inputs, actions and code are associated to the net elements. In this work we consider binary Petri nets with an interpretation that associates application code, predicates and priorities to the transitions. The firing of a transition models the execution of a piece of code. Thus we can consider three firing phases: unmarking the input places, code execution and marking output places. The priority of a transition represents the priority of the associated code in the access to the CPU and allows the effective conflict resolution among mutually excluding transitions. For the purpose of this paper, we consider that predicates over system inputs evaluate always to true.

Centralized implementation techniques [5] [7] [8] are based on two types of threads or tasks. The first type groups the tasks that implement the operational part of the system, that is, the application code associated to transitions. A CODE task is generated for each transition in the Petri net and the associated code is included. Each CODE task inherits the priority of the transition. The second type corresponds to a single task called the Coordinator, which plays the so-called token game, i.e., it makes the net evolve over time. The Coordinator establishes when transitions are enabled and must fire running the associated code. The Coordinator communicates with the CODE tasks in order to authorize their execution and has the highest priority within the implementation. The architecture is similar to an operating system in which the coordinator is the kernel and the CODE tasks are the processes executed within (see Fig. 1). Access to the processor is managed by the run-time system of the target language and by the operating system kernel based on the priorities of the CODE tasks and the Coordinator. The Coordinator is an interpreter that works over a data structure that codes the PN [8].

Apart of the simple exhaustive test of all transitions (brute force approach), there are various solutions to reduce the costs of the enabling test and then the overload introduced by the coordinator. Depending on these solutions, centralized implementations techniques can be classified into one of the following classes [9]:

Place-driven approaches. Only the output transitions of some representative marked places are tested [5]. Each transition is represented by one of its input places, the Representing Place. The rest of input places are called synchronization places. Only those transitions whose Representing Place is marked are considered as candidates for firing. The Representing Place of a transition can be static or can be dynamically selected as in [10] (in this case it is called triggering place). In the Dynamic Representing Places technique, if a Representing Place is marked but the transition is not enabled due to one or several of the synchronisation places being disabled, any one of the unmarked synchronisation places will be chosen as the new Representing Place. In this manner we can reduce the number of marked Representing Places.

Transition-driven approaches. A characterization of the enabling of transitions, different from the marking, is supplied, and only completely enabled transitions are considered. In general, an explicit representation of the marking is not necessary. This kind of technique is studied in works such as [2] or [9].

In the present work we have implemented four algorithms in which different enabled transition search techniques are developed:

- Brute Force $(B F)$.

- Enabled Transitions (ET).

- Static Representing Places (SRP)

- Dynamic Representing Places $(D R P)$

In a centralised interpreted implementation we need a static data structure which encodes the Petri net structure and a dynamic one which represents the net state or marking. In all the algorithms tested in this work we have maintained an explicit representation of the net marking in order to allow use in control applications. This implies a difference with regards to the implementation presented in [2]. All the algorithms share the same basic data structure, which the Petri net is encoded, with different access possibilities adapted to each technique. 
Program 1 presents the basic treatment cycle of the Coordinator. Here we can distinguish two phases: (1) enabling analysis and start of firing, and (2) reception of end of transition firing.

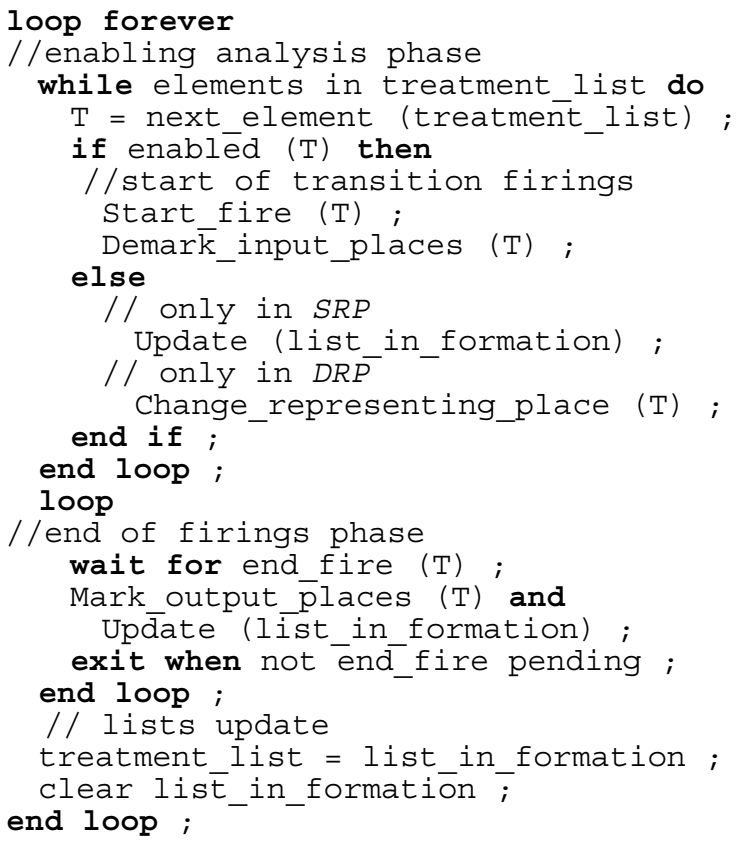

Program 1. Coordinator treatment loop

The cycle works on two lists: treatment_list and list_in_formation. The treatment_list contains the transitions which are candidates for firing, which will be analysed in the first treatment cycle phase. List_in_formation is composed throughout the treatment cycle with the transitions which will be candidates for firing in the next treatment cycle. With the use of these two lists, the net is executed by steps, avoiding the appearance of races. The fundamental difference between the implementation techniques analysed is in how the list_in_formation is formed, and hence in the transitions which are considered in each treatment cycle.

In the Brute Force technique, the treatment list is made up of all the net transitions. In the Enabled Transitions technique the following data structures will be available:

- Enabled Transitions List . Treatment list made up of the transitions with all marked input places.

- Almost Enabled Transitions List. Formation list which will be completed with the output transitions of the places marked in the firing of the transitions.

In the SRP and DRP techniques, the following data structures will be available:

- Marked Representing Places list and Marked Synchronization Places list. Treatment List with the marked Representing Places and Synchronisation Places.

- Marked Representing Places list next cycle and Marked Synchronization Places list next cycle. Formation List with the Representing Places and
Synchronisation Places marked by the firing of the transitions

In order to resolve firing conflicts between mutually exclusive transitions, the treatment list is always ordered from highest to lowest priority. In this manner, the enabled transition with the highest priority will always fire. The firing of the transition implies the un-marking of the input places (which impedes the firing of other transitions in conflict) and the sending of an authorization for the corresponding CODE task to execute its code.

Next, the coordinator enters the second execution phase, the end of the transition firings (CODE tasks complete their code), marking the output places.

In the case of the Brute Force technique, all the net transitions are contained in the treatment list and thus the list_in_formation is not necessary in this technique. In the case of Place-driven techniques, those transitions for which the Representing Place is marked are considered in the enabling test phase.

In these techniques the treatment_list is composed by the marked representing places and all represented transitions are considered as candidates for firing. If a represented transition fires, the rest of represented transitions are not tested because the representing place becomes unmarked. Finally, in the Enabled Transitions technique only those transitions which are completely enabled are contained in the treatment_list. Each time a transition fires, the transitions descending from the output places are tested and included in the list in formation if are enabled.

\begin{tabular}{|c|c|c|c|c|c|c|c|}
\hline Net & $\# \mathrm{P}$ & \#T & $f_{p}$ & $\overline{f_{s}}$ & $\overline{f f_{d}}$ & $\begin{array}{l}\text { av } \\
\text { conc }\end{array}$ & $\begin{array}{l}\text { Max } \\
\text { conc }\end{array}$ \\
\hline$S E Q$ & ne & ne & 1 & 1 & 1 & 1 & 1 \\
\hline$P A R$ & $2 * p$ & $2 * p$ & 1 & 1 & 1 & $p$ & $p$ \\
\hline PRI & $1+2 * p$ & $2 * p$ & 1.5 & 1.5 & $\frac{3 p}{1+2 p}$ & 1 & 1 \\
\hline$D B$ & $1+3 * b^{2}$ & $2 * b^{2}$ & $\frac{6 b-3}{2 b}$ & $\frac{6 b-3}{2 b}$ & $\frac{7 b-3}{4 b}$ & $\leq b-1$ & $b-1$ \\
\hline$P 1 R$ & $r+1$ & $r+1$ & $\frac{1+2 r}{1+r}$ & $\frac{1+2 r}{1+r}$ & 1 & 1 & 1 \\
\hline$P H$ & $7 * f$ & $5 * f$ & 1.6 & 1.6 & 1.14 & $\frac{f}{2}$ & $f$ \\
\hline$S Q$. & $2 r+r^{2}$ & $r+r^{2}$ & $\frac{r+2 r^{2}}{r+r^{2}}$ & $\frac{r+2 r^{2}}{r+r^{2}}$ & $\frac{r+2 r^{2}}{2 r+r^{2}}$ & $\frac{r}{2}$ & $\frac{r}{2}$ \\
\hline PR5 & $5+6^{*} p$ & $6^{*} p$ & 1.83 & 1.83 & $\frac{11 p}{5+6 p}$ & 2.5 & 3 \\
\hline
\end{tabular}

Table 1 Characterization of the Petri nets composing the net library. fp (Parallelism Factor) $=$ average number of descending places of a transition. fs (Synchronization Factor) $=$ average number of input places of a transition. fd (Descendants Factor) $=$ average number of output transitions of a place. Av. conc. $=$ average concurrence. Max conc.$=$ maximum concurrence .

\section{DESCRIPTION OF TESTS.}

As has been said, the main objective of the current paper is to evaluate the current interpreted and centralized 
implementation techniques of Petri nets from the point of view of the overload introduced for the control part. We envisage the need to carry out a series of tests with a wide range of Petri nets of different characteristics, in order to determine which algorithm is best in accordance with the type of Petri net executed. Each test consists of the execution of a Petri net with one of the implemented algorithms. The test concludes when 2000 transitions have fired, obtaining the coordinator task total computation time. Each net is also executed with the other implemented algorithms. We have implemented the centralized implementation techniques of $\mathrm{PN}$ in the Java language using the Java Real-time extension [11] and following some ideas presented in [12] [13].

The real time extension provides Java with the necessary aspects for the programming of real-time systems, e.g., pre-emptive planning based on static priorities, asynchronous transfer of control, real-time high resolution clocks, and the possibility of execution over the Java garbage collector. In our implementations, we used the Real Time Java Virtual Machine JamaicaVM v2.7 [14]. The target hardware was a personal computer with Pentium IV processor at $1.7 \mathrm{GHz}$, running Red Hat Linux 2.4.

A library of Petri nets has been developed for carrying out the tests. The library is based on eight base models which can be scaled using a parameter. Some of these models are Petri nets which are well known and frequently used in literature. The library comprises the following nets:

- SEQ. Petri nets of one sequential process with ne (1..100) states (Fig. 2.a).

- $\quad$ PAR. Petri nets of $\mathbf{p}(1 . .100)$ sequential processes of 2 places (Fig. 2.b).

- PR1. Petri nets of $\mathbf{p}(1 . .40)$ sequential processes of 2 states and a common resource (Fig. 2.c). These belong to $\mathrm{s}^{3} \mathrm{pr}$ net class [15].

- $D B$. Petri nets of b (5..11) databases (Fig. 2.d). [16].

- P1R. Petri nets of 1 sequential process and $\mathbf{r}(1 . .40)$ resources (Fig. 2.e). These belong to $\mathrm{s}^{3}$ pr net class [15].

- $P H$. Petri nets of the philosophers' problem[17] (Fig. 2.f) with f (5..40) philosophers.

- SQUARE. Petri nets of $\mathbf{r}(1 . .40)$ sequential processes of $\mathbf{r}+\mathbf{1}$ states and $\mathrm{r}$ common resources (Fig. 2.g, defined by the authors).

- PR5. Petri nets of $\mathbf{p}$ (5..62) sequential processes of 6 states and 5 common resources (not showed in figure). These belong to $\mathrm{s}^{3} \mathrm{pr}$ net class [15]. a)

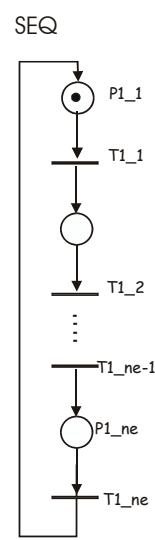

b)
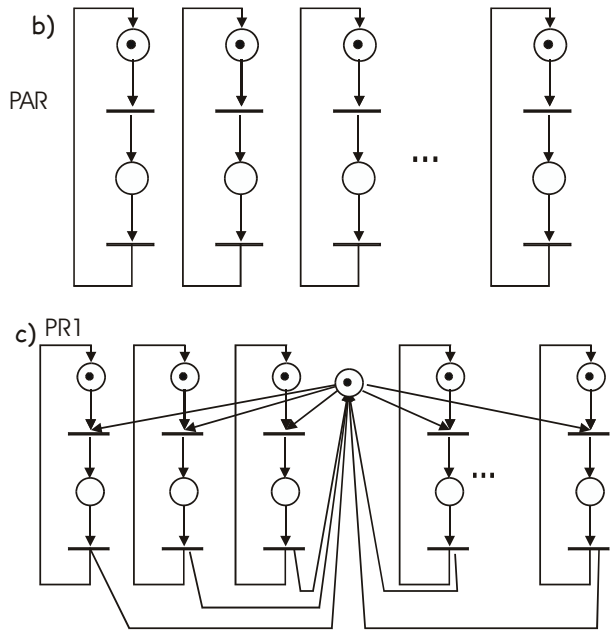

f) $\mathrm{PH}$

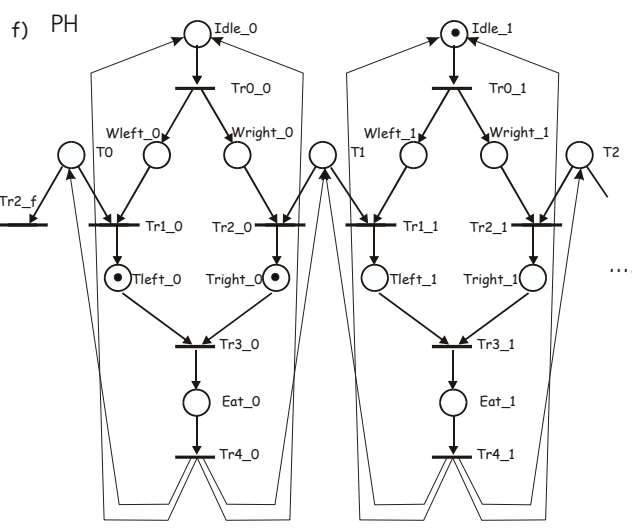

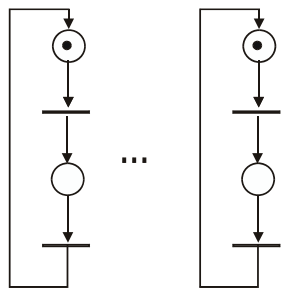

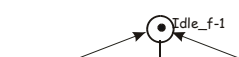

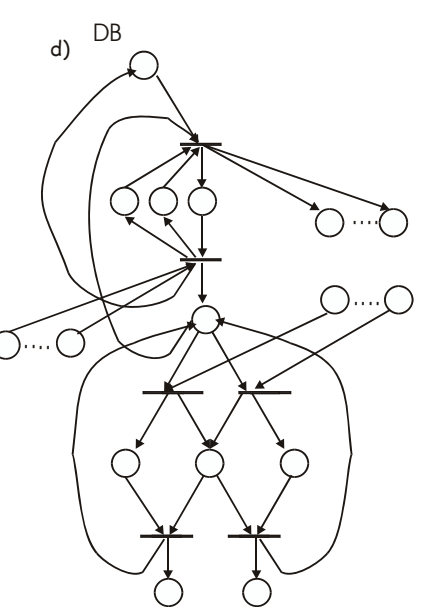

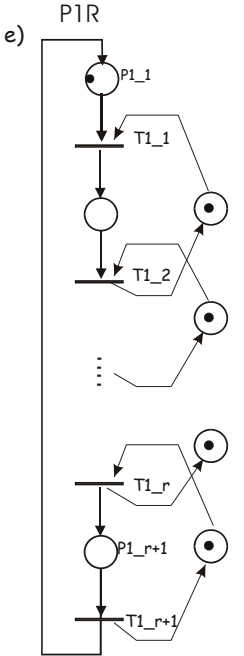

SQUARE

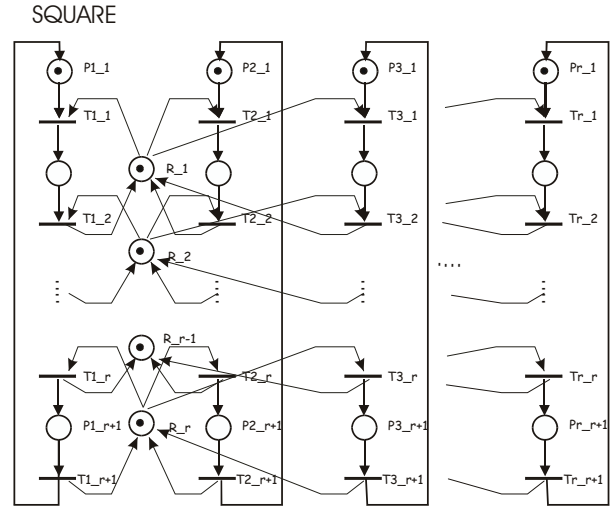

Fig. 2. Petri Nets Library 
The Petri nets belonging to the library present different characteristics in order to enable the different dynamic behaviours of the implementation techniques in studio. Hence, the nets present a different number of places and transitions, different level of concurrency, different possibilities of shared resources, different structural characteristics. These differences can be seen in Table 1, which contains some parameters [18] for the characterisation both of the structure and the behaviour of the Petri nets.

\section{PERFORMANCE ANALYSIS}

The computing time of the algorithms described in the previous section will depend on the size of the treatment and formation lists and on the number of places and transitions verified to compose these lists. The size of these lists is not static. It depends on the net structure and state and also on the sequence of events. As has been stated, in the tests carried out, the predicate or condition associated to the transition is considered true, meaning the transitions will fire immediately. The tests enables the situation of maximum overload of algorithms, since in all the cycles the maximum possible enabled transitions are fired.

Fig. 3 shows the results of the performance tests. Each plot correspond to a Petri net model of the library. The $\mathrm{X}$ axis represents the scaling parameter of the net and the $\mathrm{Y}$ axis represents the total execution time (in nanoseconds) of the Coordinator in 2000 transition firings. All graphics are titled with the name of the corresponding PN model.

Fig. 3.a shows the test results of the PR5 net model. The Brute Force algorithm only performs well for nets with few transitions. As the net size increases, the $B F$ technique increases the execution time to amounts of one order of magnitude greater than the other techniques. The fact that the enabling of all the net transitions is computed in each cycle means that the computation time is significantly greater than the other algorithms. This fact has been observed in all of the net models. Consequently, the performance analysis will be centred on the ET, SRP and DRP techniques.

Fig. 3.b shows the test results of the $S E Q$ net model. The $S E Q$ nets are sequential processes of 1 to 100 places. In each cycle there is only one place marked, and hence there is only one Representing Place marked and one enabled transition. Moreover, the transitions only have one input place, meaning the $D R P$ and $S R P$ algorithms perform similarly, since changes of Representing Places can not exist. In this test the three algorithms perform similarly and execution times depend only weakly on the size of the net.

Fig. 3.c shows the test results of the $P A R$ net model. $P A R$ nets have p sequential processes without interactions, and in each cycle there are $p$ enabled transitions that can fire simultaneously and $\mathrm{p}$ marked Representing Places. The transitions only have one input place, hence there are no Representing Place changes and the Representing Place algorithms (static and dynamic) perform similarly. In this test, the winner algorithm is ET. On the other hand, Fig. 3.d shows the test results of the $P R I$ net model. $P R I$ nets represent $\mathrm{p}$ sequential processes that are mutually excluding, thus the maximum concurrence is one. In this case, the $S R P$ and $D R P$ algorithms are the best. From these two last tests, we can conclude that the Representing Place technique is better when the net presents effective conflicts in its behaviour. As has been stated, whenever a place represents a set of transitions in conflict, if one transition fires the rest are not evaluated. In the $E T$ technique all transitions in conflict are verified.

In SQUARE and PR5 nets DRP and SRP performs well than $E T$ technique. However, in $P H$ and $D B$ nets, the $E T$ performs better. The structure of these nets are more complicated than the previous ones and it is very difficult to establish, without a behavioural study, when conflicts predominate over concurrency or vice versa. So the election of the best implementation technique depends on the net behaviour (not only on the structure). The net behaviour analysis can be performed by state space analysis (reachability graph).

As an example consider SQUARE nets. Table 1 shows that the average concurrency of SQUARE nets are $\mathrm{r} / 2$ in its behaviour. However, these nets are composed by $\mathrm{r}$ processes but the conflicts reduce the effective concurrency to half.

Table 2 shows some results of the behaviour of the implementation algorithms over some net models of the library. For ET the average size of the formation and treatment lists and the average number of tested transitions to conform the lists in each cycle are presented. In the case of $S R P$ technique, the average number of marked representing places and the average number of represented transition are presented for two different sets of representing places that we call "Bad Election" and "Good Election".

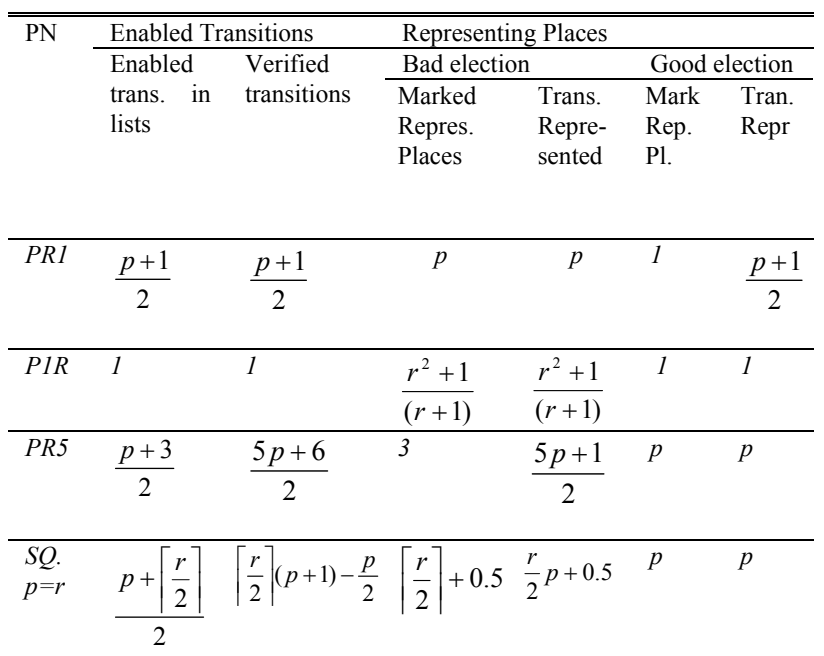

Table 2 Size of Data Structures. $r$ resources number, $\mathrm{p}$ processes number.

For example, in Enabled Transitions the tests showing poor performance for PN type PR5, PR1 or SQUARE, as seen in Table 2, are Petri nets where the number of Enabled Transitions is proportional to the number of processes, meaning the computing time of the phase of analysis of enabled transitions increases proportionally with the number of processes. 
a)

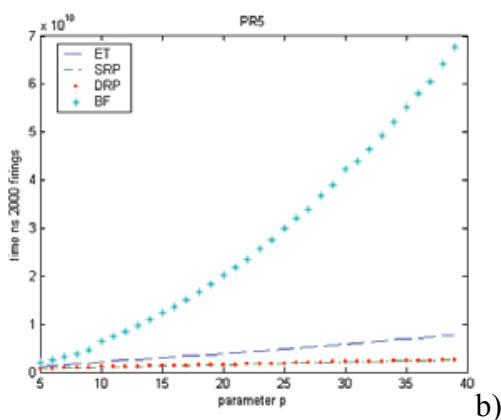

d)

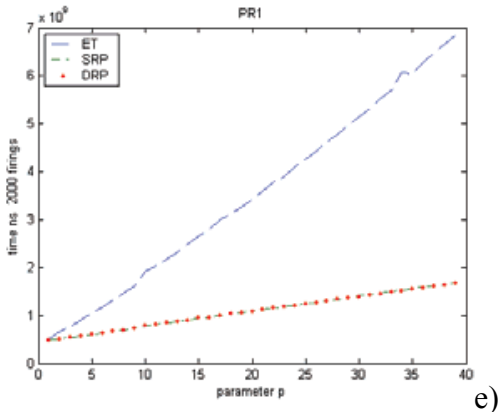

e)
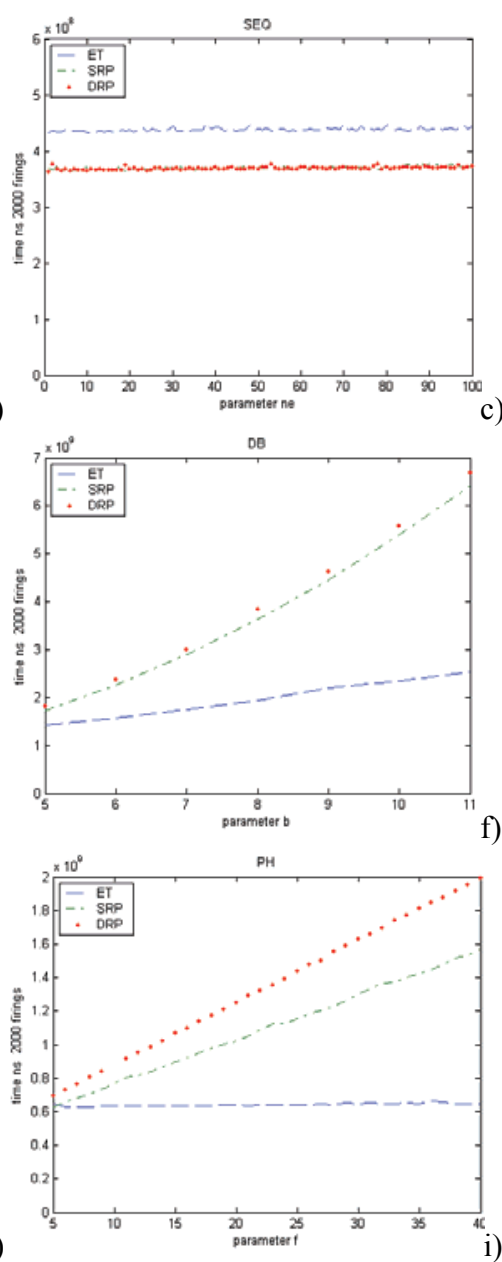

g)
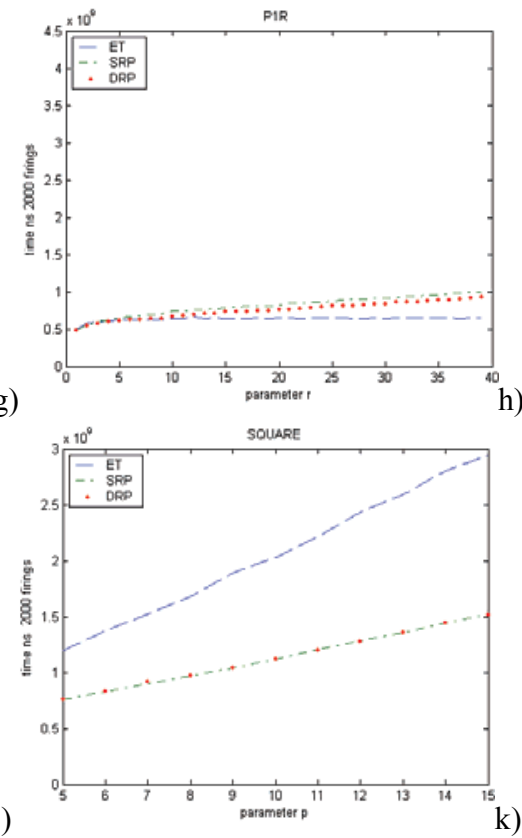

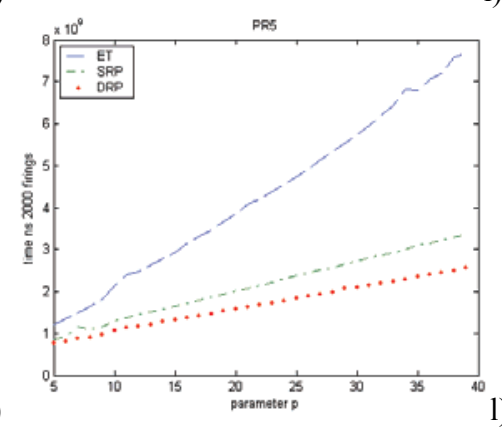

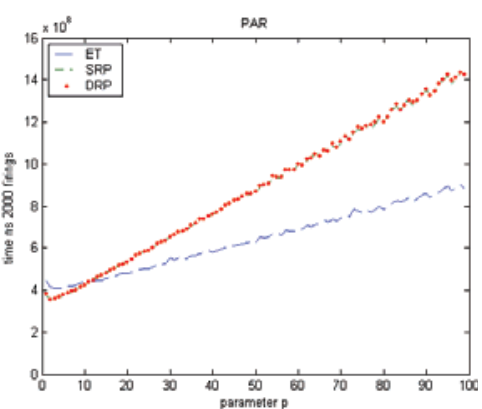
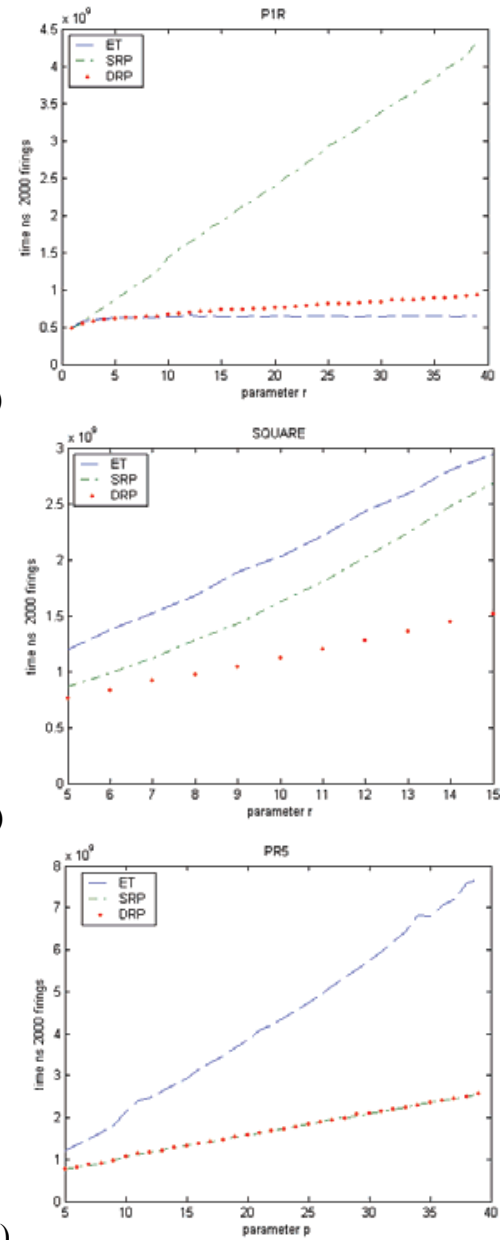

Fig. 3. Petri net tests with the $B F, E T, S R P$ and $D R P$ algorithms.

In $P R 5$ and $P R 1$ nets the number of transitions verified is proportional to the processes number. In SQUARE nets it is quadratic, meaning the lists update time increases with the number of processes. However, in the representing places technique the transitions verified (the number on represented transitions is the worst case) increases linearly. Thus, reasoning in terms of implementation data present the same results as the previous behavioural analysis.

Fig. 3.f and Fig. 3.g show the results of the $P 1 R$ net model. In the Fig. 3.f the $S R P$ technique performs worse than the others. In the $S R P$ technique the enabling test is only carried out on those transitions for which the Representing Place is marked. Obviously the efficiency of the method will depend on the choice of the Representing Places. Fig. 3.g shows the same test but with another representing place selection. The performance of the SRP improves drastically, being comparable with the other techniques. The same result can be observed for net models SQUARE and PR5.

In the test of the Fig. 3.i the winning algorithm is $D R P$. $D R P$ creates a very large set of Representing Places, choosing those of the processes, and minimises the number of transitions represented. 
a)

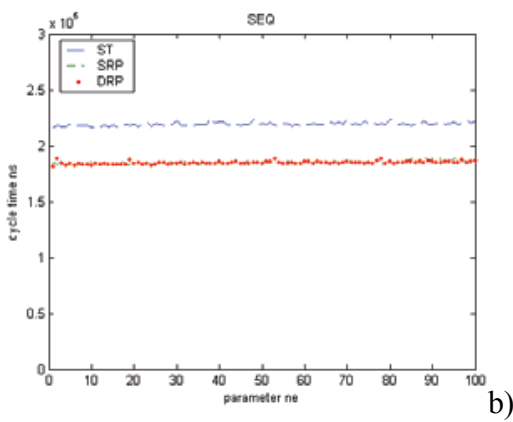

d)

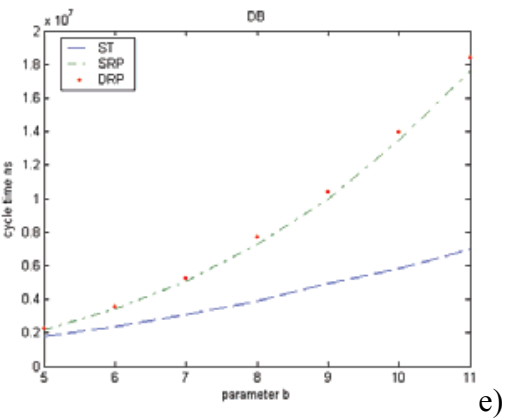

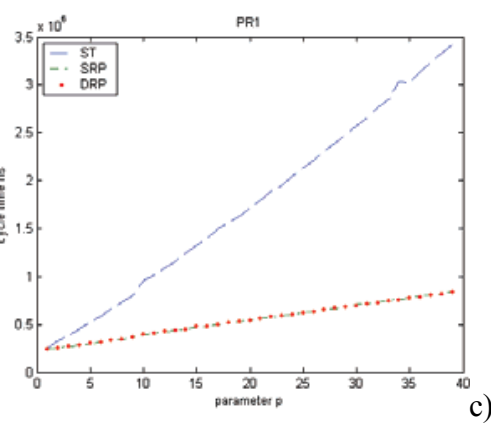

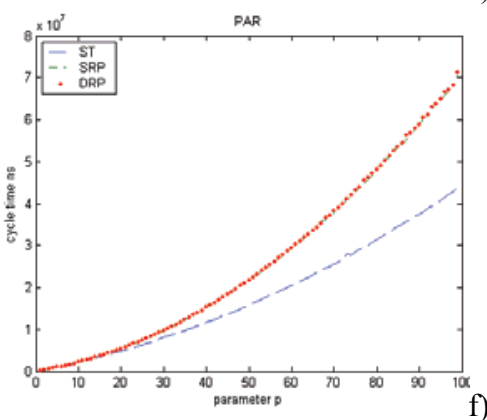

c)
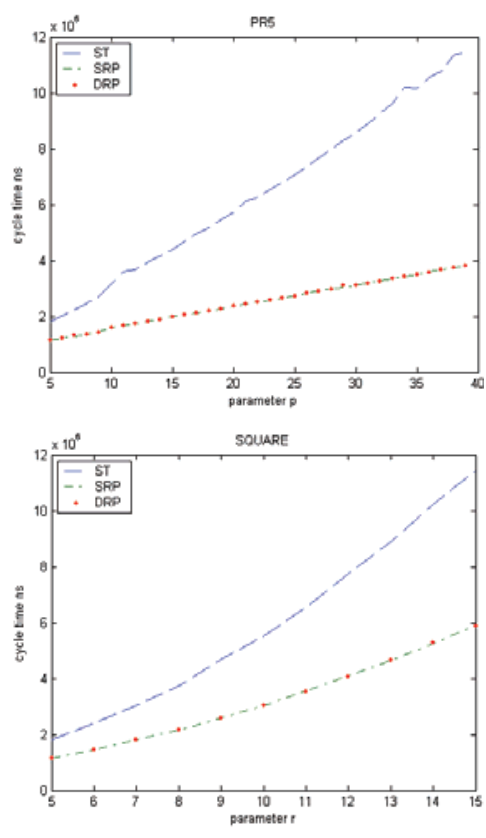

Fig. 4. Cycle time of the algorithms.

SRP minimises the number of Representing Places marked, but the number of transitions represented is greater than in $D R P$. In Fig. 3.j both algorithms $D R P$ and $S R P$ choose the same representing place selection, the $S R P$ performs equals than $D R P$.

For PR5 nets, in he test of the Fig. 3.k the winning algorithm is DRP. DRP chooses those of the processes as Representing Places, meaning there will be p Representing Places marked and $\mathrm{p}$ transitions represented. On the other hand, SRP chooses the resources amongst the Representing Places and minimises the number of Representing Places marked, but the number of transitions represented will be $2.5^{*} p+0.5$. In Fig. 3.1 both algorithms $D R P$ and $S R P$ choose the same representing place selection, the $S R P$ performs equals than $D R P$.

$D R P$ is an adaptive technique, without any a priori knowledge, directed to dynamically select the set of representing places less marked in the net evolution. However, if the net behaviour is studied a priori, it could be established the best set of places (places less marked in the time) to be used as the representing places. This set of ideal representing places [9] is that which, for any reachable marking, the transition is enabled if the Representing Place is marked If it is not possible to achieve a place which verifies this condition, fulfilment is proposed for the greatest number of reachable markings. In most cases, with a good representing place selection, the $S R P$ performs better than $D R P$ because it is not necessary to change the representing places.

Thus, we can conclude that $D R P$ does not improve the $S R P$ behaviour with an informed selection. It is concordant with the results presented in [19] that affirms that the optimization made by algorithm $D R P$ does not improve the $S R P$ behaviour appreciably.
Another interesting result of the tests is the cycle time of the Coordinator. In Petri nets with limited concurrence, for example $S E Q, P R 1$ and $P 1 R$, the maximum number of transitions that can be fired by cycle is one, or in Petri nets $P R 5$, can be fire three transitions. As it is observed in the Fig. 4.a, Fig. 4. b and Fig. 4.c the cycle time follows the same tendencies that in the graphs of the Fig. 3.

In Petri nets where the concurrence increases with the size of the Petri net as in $D B, P A R$ or SQUARE Petri nets, the maximum number of transitions that can be fired in a cycle is proportional to the size of the net and the graphs of cycle time have quadratic form (see Fig. 4.d, Fig. 4.e and Fig. 4.f). In control applications the cycle time is related to the system reaction time.

The Fig. 5 show the different control periods for the different algorithms. In the Fig. 5.a is observed the control periods for a PN PR5 with 25 processes. It is observed that if chooses algorithms $R P$, the control can be executed 17 times faster than with algorithm $B F$. If $S T$ is chosen it would execute 6 times faster than $B F$.

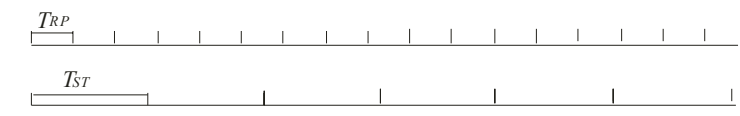

a)

$T_{B F}$

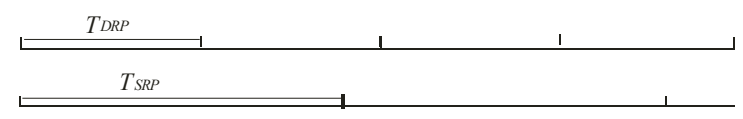

b) $\frac{T_{S T}}{\text { Fig. 5. Control Period of the algorithms: a) PR5 net with } 25 \text { processes. b) }}$ SQUARE net with 15 processes. 
The Fig. 5.b shows the results for a PN SQUARE with 15 processes. If algorithm DRP is chosen, the Petri net can be executed 2 times faster than with algorithm ST. The election of the suitable algorithm much more supposes a fast execution of the Petri net and a faster reaction of the control system.

\section{CONCLUSIONS}

In this paper we have presented a performance evaluation of interpreted and centralized implementation techniques of Petri nets. These implementation techniques allow the translation of a system model expressed by a Petri net to an actual system with the same behaviour as the model. Four techniques have been analysed: Brute Force, Enabled Transitions, Static Representing Places and Dynamic Representing Places. We have developed a library which aims to cover a wide range of Petri Nets. In accordance with the tests carried out, we determine:

- The implementation of the Enabled Transitions, Static and Dynamic Representing Places algorithms brings a drastic reduction of the execution computing time compared to the Brute Force algorithm.

- If the Static Representing Places algorithm chooses suitable Representing Places, performance is similar to or better than Dynamic Representing Places.

- The choice of the most suitable type of algorithm to execute a Petri Net depends on the Petri net behaviour (concurrency v.s. effective conflicts)..

The execution of a Petri net without a suitable algorithm can suppose great increases of computing time, and one worse and slower answer in control applications. We are currently working in a technique which allows the choice in real time of the most suitable algorithm to execute a Petri Net in accordance with its structure and the sequence of events. We also want to systematize the use of the reachability graph to select the most suitable algorithm to implement a Petri Net.

\section{ACKNOWLEDGMENTS}

We want to thanks to Santiago Velilla and Joaquin Ezpeleta for their helpful aid.

\section{REFERENCES}

[1] T. Murata. Petri nets: Properties, Analysis and Applications. Proc. of the IEEE, 77(4), 1989, 541-580.
[2] M. Silva and S. Velilla. Programmable logic controllers and Petri Nets: A comparative study. In Proc. of the Third IFAC/IFIP Symposium, Software for Computer Control1982, pages 83-88. Pergamon Press, 1982.

[3] R. Valette, M. Courvoisier, J.M. Bigou, and J. Alburkerque. A Petri net based programmable logic controller. In Proc. of IFIP Conference on Computer Applications inProduction Engineering, CAP, pages 103-116, 1983.

[4] R. David y H. Alla. Petri Nets \& Grafcet. Prentice Hall. 1992.

[5] J.M. Colom, M. Silva, and J.L. Villarroel. On software implementation of Petri nets and coloured Petri nets using high-level concurrent languages. In Proc of 7 th European Workshop on Application and Theory of Petri Nets, pages 207-241, Oxford, July 1986.

[6] D. Taubner. On the implementation of Petri Nets. In G. Rozenberg, H. Genrich, and G. Roucairol, editors, Advances in Petri Nets 1988, volume 340 of Lecture Notes in Computer Sciences, pages 418-439. Springer-Verlag, Berlin, Germany, 1988.

[7] J.L. Villarroel. Integración Informática del Control de Sistemas Flexibles de Fabricación. PhD thesis, Dpto. de Ingeniería Eléctrica e Informática, Universidad de Zaragoza, Septiembre 1990.

[8] F.J. García, J.L. Villarroel: "Modelling and Ada Implementation of Real-Time Systems using Time Petri Nets." Proc. of the 21 st IFAC/IFIP Workshop on Real-Time Programming. Gramado - RS, Brazil. November 1996.

[9] J.L. Briz. Técnicas de implementación de redes de Petri. PhD thesis, Univ. Zaragoza, 1995.

[10] R. Valette and B. Bako. Software implementation of Petri Nets and compilation of rule-based systems. In 11th International Conference on Application and Theory ofPetri Nets, pages 27-29, Paris, France, June 1990.

[11] The Real-Time for Java Expert Group. The Real-time Specification for Java. https://rtsj.dev.java.net/. Addison Wesley, 2000.

[12] R. Piedrafita and J.L. Villarroel. Petri nets and Java. Real-Time Control of a flexible manufacturing cell. . 11th IEEE International Conference on Emerging Technologies and Factory Automation. Prague, September 2006 .

[13] R. Piedrafita and J.L. Villarroel. Implementation of Time Petri Nets in Real-time Java. The 4th International Workshop on Java Technologies for Real-time and Embedded Systems - JTRES 2006. Paris, October 2006 .

[14] Aicas GmbH. JamaicaVM Realtime Java Technology. http://www.aicas.com/jamaica.html

[15] J. Ezpeleta and J.M. Colom and J. Martínez: "A Petri net based deadlock prevention policy for flexible manufacturing systems". IEEE Transactions on Robotics and Automation, Vol. 11, N. 2, pp. 173-184, April 1995

[16] K. Jensen. "Coloured petri nets". In W. Brauer, W. Rdsig, and G. Rozenberg, editors, Petri Nets: Central Models and Their Properties, pages 248-299. Springer-Verlag, 1987. LNCS 254.

[17] Dijkstra E. W.: Hierarchical ordering of sequential processes. Acta Informática, vol.1, pp. 111-127

[18] S. Velilla. Lógica Programada y Redes de Petri. PhD thesis, Dpto. de Ingeniería Eléctrica e Informática, Universidad de Zaragoza, 1987.

[19] B. Bako. Mise en oeuvre et simulation du niveau coordination de la commande des ateliers flexibles: une approche mixte reseaux de Petri et systemes de regles. PhD thesis, Universite Paul Sabatier de Toulouse, 7, avenue du Colonel Roche, 31077 Toulouse, Cedex, France, 1990. 